\title{
Pulmonalembolie und direkte orale Antikoagulantien
}

\author{
Horst Olschewski
}

Eingegangen: 21. Januar 2016 / Angenommen: 25. Februar 2016

(C) Die Autor(en) 2016. Dieser Artikel ist auf Springerlink.com mit Open Access verfügbar.

Zusammenfassung Für die Diagnostik und Therapie der venösen Thromboembolie wurde 2014 eine aktuelle Europäische Leitlinie publiziert. Danach werden wie bisher Hochrisiko- und Niedrigrisiko-Lungenembolien unterschieden. Neu ist die Definition von Lungenembolien mit intermediärem Risiko. Das Risiko entscheidet über das diagnostische und therapeutische Vorgehen.

Für die akute Therapie standen früher fast nur Heparinoide zur Verfügung, die nach einigen Tagen auf einen Vitamin K Antagonisten umgestellt wurden. Mit den direkten oralen Antikoagulantien (DOAK) wurde eine Medikamentengruppe verfügbar, die einen Wirkungseintritt wie ein Heparinoid haben und übergangslos als orale Dauermedikation verfügbar sind. Anders als Vitamin K Antagonisten sind keine wiederholten Gerinnungskontrollen nötig. Die Dosierung ist unkompliziert, aber nicht ganz selten sind Abweichungen von der Standarddosis erforderlich. Der folgende Artikel versucht eine rasche Orientierung zu bieten.

Schlüsselwörter Lungenembolie · Antikoagulation · Thrombolyse $\cdot$ DOAK

\section{Pulmonary embolism and direct oral anticoagulants}

Summary A new European guideline for the diagnostics and therapy of venous thromboembolism has been published in 2014. As previously, high-risk and low-risk

Univ Prof. Dr. med. H. Olschewski $(\bowtie)$

Division of Pulmonology, Department of Internal Medicine,

LKH University Hospital Graz - Medical University of Graz,

Auenbruggerplatz 15,

Graz 8036, Österreich

E-Mail: horst.olschewski@medunigraz.at pulmonary embolism is distinguished, however, in the new guideline there is also a definition of intermediate risk $P E$. The risk finally decides about the diagnostic and therapeutic procedure.

Previously, nearly only heparinoids were available for therapy of acute venous thromboembolism and after some days they were switched to a vitamin $\mathrm{K}$ antagonist. The direct oral anticoagulants (DOAC) represent a group of drugs that start working as rapidly as heparinoids and can be applied as long-term oral medication. In contrast to vitamin $\mathrm{K}$ antagonists, repeated coagulation tests are not necessary. Dosing is easy, although in quite a few cases dose adaptations compared to the standard dose may be necessary. The following article tries to give a rapid orientation.

Keywords Pulmonary embolism • Anticoagulation . Thrombolysis · Direct oral anticoagulants

\section{Pulmonalarterienembolie}

Für die Pulmonalarterienembolie wurde 2014 eine neue Leitlinie der europäischen kardiologischen Gesellschaft (ESC) in Kooperation mit der europäischen pneumologischen Gesellschaft (ERS) herausgegeben [1]. Diese wurde durch eine Vielzahl neuer Studiendaten untermauert. Das führte unter Anderem dazu, dass die Schweregradeinteilung der Lungenembolie revidiert wurde. Auf Basis der aktuellen Evidenz unterscheiden wir jetzt Lungenembolien mit hohem Risiko, intermediär hohem Risiko, intermediär niedrigem Risiko und niedrigem Risiko.

Die Hochrisiko Lungenembolie erkennt man am kardiogenen Schock. Sie stellt nach wie vor eine klare Indikation zur sofortigen Thrombolysetherapie dar. Alternativ kann eine Katheterfragmentation oder eine 
Embolektomie in Frage kommen, wenn die strukturellen Voraussetzungen erfüllt sind.

Die Niedrigrisiko Lungenembolie hat definitionsgemäß ein so niedriges Mortalitätsrisiko, dass es möglich ist, diese Patienten vom Anfang an ambulant zu behandeln. Man erkennt sie an einem sPESI von 0 . Die Bezeichnung „sPESI“ steht für den simplified PESI (pulmonary embolism severity index). Es handelt sich um ein validiertes Instrument zur Abschätzung der Mortalität bei akuter Lungenembolie [2, 3]. Die Faktoren Alter $>\mathbf{8 0}$ Jahre, aktives Krebsleiden, chronische Herzoder Lungenkrankheit, Puls $>110 / \mathrm{min}$, systolischer Blutdruck $<100 \mathrm{mmHg}$, Sauerstoffsättigung $<90 \%$ zählen jeweils einen Punkt. Wenn der Patient keinen dieser Faktoren hat, so beträgt sein sPESI 0 Punkte. Der sPESI ist sehr gut geeignet für die Kitteltasche und auch einfach zu merken.

Die Intermediär-niedrig Risikogruppe soll stationär aufgenommen werden, wenn auch nicht primär auf die Intensivstation. Solche Patienten erkennt man daran, dass sie entweder eine normale rechtsventrikuläre Funktion oder ein normales BNP bzw. Troponin haben.

Bei der Intermediär-hoch Risikogruppe muss der Patient in Lysebereitschaft überwacht werden. Das heißt in aller Regel: Aufnahme auf der Intensivstation. Diese Patienten erkennt man daran, dass sie eine rechtsventrikuläre Dilatation und ein erhöhtes BNP bzw. Troponin haben.

Nach überstandener Lungenembolie muss jeder Patient antikoaguliert werden. Lag der Lungenembolie ein transientes Risiko zugrunde, welches jetzt nicht mehr besteht, so genügt eine Antikoagulation von 3 Monaten. Liegt dagegen ein persistierendes Thromboembolierisiko vor, so wird eine längerfristige Antikoagulation empfohlen, sofern es keine Kontraindikationen dagegen gibt. Die Leitlinie ermahnt aber an mehreren Stellen, im langfristigen Verlauf immer wieder neu eine Abschätzung von Nutzen und Risiko der Antikoagulation vorzunehmen.

Wenn man sich unsicher ist, ob ein persistierendes oder ein transientes Risiko für die Thromboembolie vorlag, so kann man sich den D-Dimer Test zu Hilfe nehmen. Vier Wochen nach Absetzen der Antikoagulation wird das D-Dimer gemessen. Wenn es erhöht ist, hat man ein starkes Argument, die Antikoagulation länger fortzusetzen. Ist es normal, kann man in Erwägung ziehen, die Antikoagulation endgültig abzusetzen. Eine aktuelle Studie hat festgestellt, dass das Rezidivrisiko bei diesen Patienten noch immer inakzeptabel hoch sein kann, insbesondere bei Männern [4].

Patienten mit einer zweiten venösen Thromboembolie sollten jedenfalls lebenslang antikoaguliert werden, sofern es keine starken Gegenargumente gibt. Ansonsten ist das Risiko für wiederholte thromboembolische Ereignisse als sehr hoch einzuschätzen. Der Stellenwert von Thrombophiliefaktoren in der plasmatischen Gerinnung (Faktor V Leiden, etc.) in den Guidelines ist derzeit sehr gering. Auch in der Schwangerschaft sind solche
Faktoren nicht wirklich hilfreich für die Entscheidung für oder gegen eine Antikoagulation [5]. Ausnahme ist ein hochtitriges Lupus Antikoagulans.

\section{Diagnostik}

Der Goldstandard zum Nachweis einer akuten pulmonal arteriellen Embolie ist das Computertomogramm (CT) in Form einer CT-Pulmonalisangiographie (CTPA). Diese Untersuchung hat eine sehr gute Spezifität und Sensitivität. Nur wenn es aufgrund der anamnestischen Angaben und der klinischen Befunde eher unwahrscheinlich ist, dass eine Lungenembolie vorliegt, so sollte zunächst der D-Dimer Test durchgeführt werden. Wenn der dann negativ ist, kann getrost auf eine weitere Abklärung mittels CT verzichtet werden, denn das Risiko für das Vorliegen einer Lungenembolie ist denkbar gering. Ist er positiv, so muss die CTPA erfolgen. Deuten die Anamnese oder der klinische Befund darauf hin, dass tatsächlich eine Lungenembolie vorliegt (mittlere oder hohe Vortestwahrscheinlichkeit), so ergibt das D-Dimer keinen Sinn. Stattdessen wird von vornherein eine CTPA empfohlen. Bei Kontrastmittelunverträglichkeit oder Niereninsuffizienz ist dann die Perfusionsszintigraphie die Methode der Wahl zum Ausschluss einer Lungenembolie. Das gilt auch in der Schwangerschaft. Wenn das native Röntgenbild normal ist, aber die Perfusionsszintigraphie einen typischen Befund zeigt, dann gilt das als Nachweis der Embolie und eine Ventilationsszintigraphie ist nicht erforderlich. Allerdings kann hierbei auch eine chronisch thromboembolische pulmonale Hypertonie vorliegen, die eine seltene aber wichtige Differentialdiagnose der akuten Lungenembolie im Szintigramm darstellt. Ist eine venöse Thromboembolie eher unwahrscheinlich (niedrige Vortestwahrscheinlichkeit) und das D-Dimer ist niedrig, so kann auf eine CTPA und auf eine Antikoagulation verzichtet werden.

\section{DOAK's}

Die direkten oralen Antikoagulantien stellen eine der wichtigsten pharmakologischen Innovationen der letzten Jahre dar. Sie haben im Wesentlichen die Wirkungen wie ein Heparinoid, werden aber als Tabletten eingenommen. Die Wirkung setzt schnell ein und hat eine im Vergleich zu Vitamin K Antagonisten kurze Halbwertszeit. Weder Spiegelkontrollen noch funktionelle Kontrollen der Gerinnung sind erforderlich. Die Datenevidenz für den Vergleich zwischen DOAKs und konventionellen Antikoagulantien wie Marcumar oder Sintrom zeigte, dass die DOAKs den konventionellen Medikamenten keinesfalls unterlegen sind, sofern es sich um die Indikationen akute Lungenembolie, Rezidivprophylaxe und Langzeitrezidivprophylaxe handelt. Das gleiche gilt für das nicht-valvuläre Vorhofflimmern und die entsprechende Apoplex Prophylaxe. Dies ist im 
Tab. 1 Aktuell in Österreich zugelassene DOAKs in der Indikation akute venöse Thromboembolie und Rezidivprophylaxe.

\begin{tabular}{|c|c|c|c|c|}
\hline Substanz & Handelspräparat & Anfangsdosis bei akuter VTE & Langzeitdosis & Dosisadaptation \\
\hline Dabigatran & Pradaxa ${ }^{\circledR}$ & Heparinoid für 5 Tage & $2 \times 150 \mathrm{mg}$ & Alter $>80 \mathrm{~J} ;$ GFR $<50 \mathrm{ml} / \mathrm{min}^{\mathrm{a}} ;$ P-gp Inhibitoren ${ }^{\mathrm{b}}$ \\
\hline Rivaroxaban & Xarelto ${ }^{\circledR}$ & $2 \times 15 \mathrm{mg}$ für 3 Wochen & $1 \times 20 \mathrm{mg}$ & $\begin{array}{l}\text { GFR }<30 \mathrm{ml} / \mathrm{min}^{\mathrm{c}} ; \text { Vorsicht bei Cyp3A4 und P-gp } \\
\text { Inhibitoren und Induktoren }{ }^{d}\end{array}$ \\
\hline Apixaban & Eliquis $^{\circledR}$ & $2 \times 10 \mathrm{mg}$ für 7 Tage & $2 \times 5 \mathrm{mg}$ bis 6 Mon, dann $2 \times 2,5 \mathrm{mg}$ & $\mathrm{Cr}>1,5 \mathrm{mg} \%{ }^{\mathrm{d}, \mathrm{e}, \mathrm{f}} ;$ Alter $>80 \mathrm{~J} ; \mathrm{KG}<60 \mathrm{Kg}$ \\
\hline Edoxaban & Lixiana $^{\circledR}$ & Heparinoid für 5 Tage & $1 \times 60 \mathrm{mg}$ & $\mathrm{GFR}<50 \mathrm{ml} / \mathrm{min}^{\mathrm{c}} ; \mathrm{KG}<60 \mathrm{~kg} ;$ P-gp Inhibitoren ${ }^{\mathrm{b}}$ \\
\hline \multicolumn{5}{|c|}{ 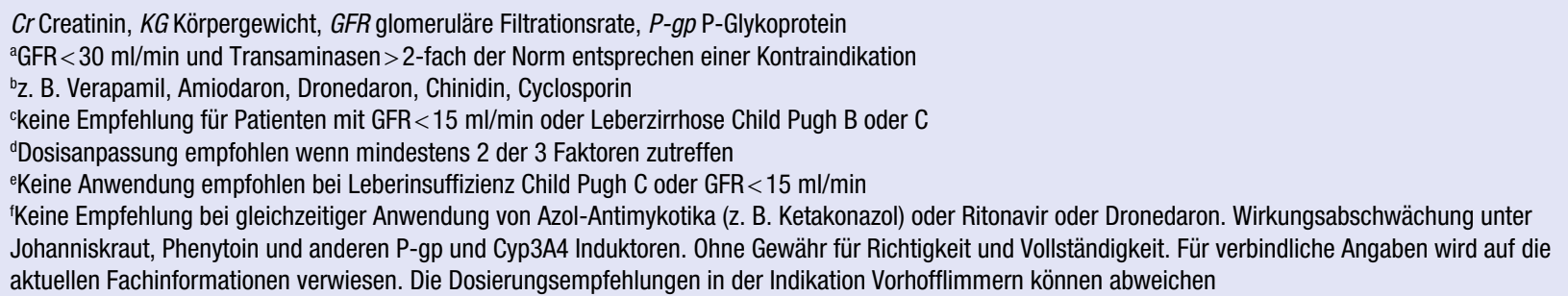 } \\
\hline
\end{tabular}

Übrigen die mit Abstand häufigste Indikation für DOAKs. Dagegen sind die DOAKs nach bisherigem Kenntnisstand für das valvuläre Vorhofflimmern und die VTE Prophylaxe bei künstlichen Herzklappen nicht geeignet. Diese Patienten brauchen also weiterhin einen Vitamin K Antagonisten, ebenso wie Dialysepatienten und Kinder.

Seitens der Nebenwirkungen stehen Blutungen im Vordergrund, wie erwartet. Allerdings gibt es hier Vorteile gegenüber den Vitamin K Antagonisten, insbesondere bezüglich intrazerebraler Blutungen. Auch bei akuten Notfalloperationen oder bei Trauma scheinen DOAK vorteilhaft gegenüber Vitamin K Antagonisten zu sein.

Praktische Überlegungen Bei den DOAKs unterscheiden wir Faktor II und Xa Antagonisten. Das macht aber keinen prinzipiellen Unterschied hinsichtlich der Handhabung. Für jede einzelne Substanz gibt es ein eigenes Dosierungsschema. Meist gilt eine etwas höhere Dosierung für die ersten Wochen und dann eine etwas niedrigere Dosis für die Langzeittherapie oder die Therapie wird mit einem Heparinoid eingeleitet und dann mit dem DOAK fortgesetzt. Die Präparate werden nach Dosierungsanweisung einmal oder zweimal täglich eingenommen. Die Wirkung setzt wenige Stunden nach der Applikation ein und nach einer Pause von 24-48 Stunden (substanzabhängig) sind wieder operative Eingriffe möglich. Insofern gelten für die DOAKs ähnliche Regeln, wie für ein fraktioniertes Heparinoid. In seltenen Einzelfällen kann es bei den Xa Antagonisten indiziert sein, den Faktor Xa Spiegel zu messen. Der Nutzen solcher Messungen wird aber kontrovers diskutiert.

Allgemein wird die Anwendung von DOAKs nicht empfohlen bei Überempfindlichkeit gegen den Wirkstoff, Schwangerschaft und Stillzeit, klinisch relevanter Blutung oder Koagulopathie, gastrointestinaler ulcerativer Erkrankung und bakterieller Endokarditis. Vorsicht ist geboten bei gleichzeitiger Anwendung von anderen Antikoagulantien oder Thrombozytenaggregationshemmern. Wenn bei einer akuten Lungenembolie noch nicht absehbar ist, ob eine Lysetherapie notwendig sein wird, sollte kein DOAK eingesetzt werden, sondern unfraktioniertes Heparin. Die Anwendung von DOAKs bei Tumorpatienten wurde nicht ausreichend geprüft und wird daher nicht offiziell empfohlen. Das Gleiche gilt für Kinder unter 18 Jahren. Tab. 1 gibt eine Übersicht über die zugelassenen DOAKs in der Indikation „venöse Thromboembolie und Rezidivprophylaxe“ und die verschiedenen Faktoren, die zu beachten sind, wenn es um eine Dosisreduktion oder eine Kontraindikation geht.

\section{Fazit}

Die ESC/ERS Leitlinien machen klare Vorgaben für das diagnostische und therapeutische Vorgehen bei einer akuten Lungenembolie und für die mittel- und langfristige Thromboembolieprophylaxe. Die direkten oralen Antikoagulantien (DOAKs) stellen eine wichtige Therapieoption dar, die einerseits die Heparinoide und andererseits die Vitamin K Antagonisten ersetzen können. Sie sind trotz der vielen und teils komplexen Regeln zur Dosisadaptation relativ einfach zu handhaben und zeichnen sich gegenüber einer konventionellen Therapie mit Vitamin K Antagonisten durch gleiche bis erhöhte Sicherheit bei gleicher bis erhöhter Effektivität aus.

\section{Open Access}

Dieser Artikel unterliegt den Bedingungen der Creative Commons Attribution License. Dadurch sind die Nutzung, Verteilung und Reproduktion erlaubt, sofern der/die Originalautor/en und die Quelle angegeben sind.

\section{Einhaltung ethischer Richtlinien}

\section{Interessenkonflikt}

H. Olschewski erklärt, dass kein Interessenkonflikt besteht. 


\section{Literatur}

1. Konstantinides SV, et al. ESC guidelines on the diagnosis and management of acute pulmonary embolism. Eur Heart J. 2014;35:3033-69k.

2. Jimenez D, et al. Simplification of the pulmonary embolism severity index for prognostication in patients with acute symptomatic pulmonary embolism. Arch Intern Med. 2010;170:1383-9.

3. Jimenez D, et al. Prognostic significance of deep vein thrombosis in patients presenting with acute symptomatic pulmonary embolism. Am J Respir Crit Care Med. 2010;181:983-91.
4. Kearon C, et al. D-dimer testing to select patients with a first unprovoked venous thromboembolism who can stop anticoagulant therapy: a cohort study. Ann Intern Med. 2015;162:27-34.

5. Rodger MA, et al. Antepartum dalteparin versus no antepartum dalteparin for the prevention of pregnancy complications in pregnant women with thrombophilia (TIPPS): a multinational open-label randomised trial. Lancet. 2014;384:1673-83. 